\title{
Effects of chronic remote ischemic conditioning on atrial fibrillation burden in patients with permanent pacemakers
}

\section{Long Chen}

Southeast University Zhongda Hospital https://orcid.org/0000-0003-4894-6658

Yaowu Liu

Zhangda Hospital, Southeast University

Didi Zhu

Southeast University Zhongda Hospital

Jinbo Yu

Southeast University Zhongda Hospital

\section{Chunlei Yu}

Zhangda Hospital, Southeast University

\section{Zhongpu Chen}

Zhangda Hospital, Southeast University

\section{Hong Jin}

Southeast University Zhongda Hospital

\section{Liqun Ren}

Zhangda Hospital, Southeast University

Qianxing Zhou ( $\sim$ longchen@seu.edu.cn )

Zhangda Hospital, Southeast University https://orcid.org/0000-0002-8469-5676

\section{Research article}

Keywords: chronic remote ischemic conditioning, atrial fibrillation, atrial high-rate episodes, pacemaker

Posted Date: August 26th, 2020

DOl: https://doi.org/10.21203/rs.3.rs-57419/v1

License: (c) (1) This work is licensed under a Creative Commons Attribution 4.0 International License. Read Full License 


\section{Abstract}

Background: The burden of atrial fibrillation (AF) has been attractive recently and may be associated with stroke risk and mortality. Remote ischemic conditioning could reduce the incidence, inducibility and sustainability of AF. But the effects of chronic remote ischemic conditioning (CRIC) on atrial fibrillation burden in patients with pacemaker implanted are still unknown.

Design: This was a single-center, prospective, randomized, open-labeled clinical study.

Methods: Sixty-six patients with permanent pacemakers were randomly divided into CRIC group and control group after 4 weeks screening. CRIC treatment was performed twice a day for 12 weeks. A remote ischemic conditioning protocol consisted of $4 \times 5 \mathrm{~min}$ inflation/deflation of the blood pressure cuff applied in the upper arm to create intermittent arm ischemia. Pacemaker programming was performed for four times: before screening (-4-week), after screening and before randomization (0-week, baseline), 4week and 12-week follow-up. Data including AF burden, longest duration of AF and cumulative numbers of atrial high-rate episodes (AHREs) were collected.

Results: Sixty-one patients (31 patients in CRIC group and 30 patients in control group) completed the study. CRIC was well-tolerated by patients after 12 weeks treatment. The burden of AF in CRIC group was decreased significantly at 4 -week compared with that at 0 -week $(14.7 \% \pm 18.5 \%$ vs $17.0 \% \pm 20.7 \%, p<$ $0.001)$, which further decreased at 12 -week compared with that at 0 -week $(8.6 \% \pm 10.2 \%$ vs $17.0 \% \pm 20.7 \%$, $p<0.001)$ and that at 4 -week $(8.6 \% \pm 10.2 \%$ vs $14.7 \% \pm 18.5 \%, p<0.001)$, which was not observed in the control group. AF burden also reduced significantly after 12-week CRIC compared with that in control group $(8.6 \% \pm 10.2 \%$ vs $17.6 \% \pm 19.5 \%, p=0.013)$. Repeated measurement ANOVA showed that the changes of AF burden was associated with CRIC instead of time $(p<0.01)$. In addition, there were trends that longest duration of AF and cumulative numbers of AHREs were reduced after 12-week CRIC.

Conclusions: This study suggests that a 12-week course of CRIC treatment could reduce AF burden in patients with permanent pacemakers, supporting widespread use of CRIC in the daily lives of these patients, which need to be verified in the future.

\section{Clinical Trial Registration}

Registration name: Effects of remote ischemic conditioning on atrial fibrillation burden in patients with paroxysmal atrial fibrillation

Registration number: ChiCTR-IOR-17012666

Registered 14 September 2017; URL: www.chictr.org.cn/showproj.aspx?proj=21591

\section{Introduction}


Atrial fibrillation (AF) is the most common persistent arrhythmia, independently associated with a twofold increased risk of all-cause mortality in women and a 1.5 -fold in men ${ }^{1}$. AF is also related to higher morbidity of stroke, and prevention of stroke can prolong the patient's life $\mathrm{e}^{12}$. The exact mechanism of AF is very complicated and has not been fully elucidated yet, which may include changes of the extracellular matrix, ion channel, fibroblast, fat cells, myocyte, autonomic nervous system, etc ${ }^{1}$. AF burden, the percentage of AF lasting time, which is usually detected as atrial high-rate episodes (AHREs) by cardiac implanted electronic devices (CIEDs) ${ }^{3}$, is not a major factor in decision on the choice of an intervention in most guidelines ${ }^{14}$. However, some studies recently have shown that AF burden was associated with stroke risk ${ }^{5}$ and mortality ${ }^{7}$, suggesting that lowering AF burden may decrease the risk of stroke and improve the prognosis.

Remote ischemic conditioning (RIC), induced by repeated, transient and non-lethal ischaemia in a distant tissue, has been attractive as it can provide protection of another organ from damage, which could be generated intra-organ ${ }^{8}$ and inter-organ ${ }^{9}$. Animal studies have shown that RIC of different organs such as kindey, liver, mesentery and limbs, could make the heart escaped from ischemia/ reperfusion damage ${ }^{9} 10$. Some clinical studies followed have confirmed the cardioprotective effects of RIC, which usually consisted of 3 or 4 cycles of intermittent inflation/ deflation of a blood pressure (BP) cuff applied to the arm or leg. It was demonstrated that RIC could improve both short-term ${ }^{11}$ and long-term ${ }^{12}$ clinical outcomes in patients with ST-segment elevation myocardial infarction. Recently, a meta-analysis pooling the results of 30 trials of coronary artery bypass graft (CABG) or valve surgery found that RIC reduced postoperative troponin release compared to the control arm, with no effects on the incidence of acute kidney injury, acute myocardial infarction, and mortality ${ }^{13}$.

Long-term repeated RIC, now called chronic RIC $(\mathrm{CRIC})^{14}$, may increase the 'dose' of RIC and subsequently lead to enhanced protection ${ }^{15}$. It was showed that RIC before CABG surgery and/or valve surgery could reduce the incidence of postoperative AF by $54 \%(11 \% \text { RIC vs } 24 \% \text { control; } p=0.031)^{16}$. Kosiuk et al recently found RIC reduced the inducibility and sustainability of paroxysmal AF (PAF), which was probably mediated by changes in electrophysiological properties of the atria ${ }^{17}$. But the effect of CRIC on AF burden is still unknown. Our previous study ${ }^{18}$ showed that 6 -week CRIC could improve heart rate variability (HRV) in mild stable ischemic heart failure, which suggested CRIC may have potential benefits on heart rhythm by modifying stress of autonomic nervous system ${ }^{19}$. This study aimed to examine whether CRIC can decrease the AF burden or not in patients with CIED.

\section{Methods}

\section{Ethics Statement}

This study was approved by the ethics committee of Zhongda Hospital, Southeast University (Nanjing, China). All the subjects provided a full, informed, written consent before entering the study. 


\section{Study population}

Patients with dual chamber permanent pacemaker (Medtronic) were recruited (by $\mathrm{YJ}$ ), who received programming in the outpatient department of Cardiology in Zhongda Hospital since Aug 2017. Patients enrolled before 4-week screening should fulfill the following criteria: (1) pacemaker (DDD, Medtronic Inc., Minnesota, USA) with high sensitivity and specificity for the detection of AF or AHREs has been implanted for at least 3 months; (2) atrial sensitivity was more than $1.5 \mathrm{mV}$ with bipolar sensing; (3) the burden of AF was $\geq 1 \%$ and $\leq 70 \%$.

The exclusion criteria were as follows: (1) persistent or permanent AF (longest AF duration $\geq 168$ hours); (2) previous history of atrial flutter or atrial tachycardia (except those being successfully ablated); (3) comorbidities related to AF, including hyperthyroidism, pericarditis, myocarditis or pulmonary embolism, etc.; (4) pacemaker and / or pacemaker leads dysfunction; (5) pacemaker need to be replaced in the near future; (6) recent (within 3 months) acute coronary syndromes, percutaneous coronary intervention, CABG or stroke; (7) severe heart valve disease; (8) uncontrolled hypertension (systolic BP > $160 \mathrm{mmHg}$ or diastolic BP > $100 \mathrm{mmHg}$ ); (9) peripheral arterial disease; (10) active cancer; and (11) presence of other serious systemic diseases.

After 4-week screening, the patients should fulfill the following criteria in pacemaker programming before randomization: (1) the burden of AF was $\geq 2 \%$ and $\leq 70 \%$; (2) the burden variety of $A F$ was less than $15 \%$ compared with that before screening.

\section{Study Design}

This was a single-center, prospective, randomized, open-labeled clinical study, and the flow chat is illustrated in Fig. 1. Patients included in the study after 4-week screen were randomly divided into two groups (assigned by $\mathrm{CZ}$ ) at 1:1 ratio in accordance with the random number table (allocation sequence generated by ZD): 1) Control group, in which patients received regular follow-up without CRIC; 2) CRIC group, in which patients received 12-week CRIC treatment. The baseline data were collected just before randomization. Follow-up visits were scheduled at baseline, 4 weeks, and 12 weeks. CRIC was performed as described elsewhere before ${ }^{18}$. Briefly, a BP cuff was applied to the left upper arm of the patient. BP was measured firstly, and then the BP cuff was inflated to a pressure of $20 \mathrm{mmHg}$ greater than the patient's systolic BP for 5 minutes. Afterwards, the cuff was deflated. Five minutes later, the cuff was inflated and deflated again as before. A RIC protocol applied consisted of $4 \times 5 \mathrm{~min}$ inflation/ deflation of the cuff. Two physicians confirmed that the subjects operated the RIC procedure correctly in the first week. CRIC treatment was then repeated each morning and evening mostly by the patients themselves.

According to our preliminary study, we hypothesized AF burden decreased by $10 \%$ in CRIC group compared with that in control group, thus 30 patients in each group are necessary to offer $80 \%$ power at 0.05 significant level according to $1: 1$ ratio of random grouping. Anticipating $10 \%$ of patients potentially non-assessable on 3-month study, 66 randomized patients in total are needed. 


\section{Pacemaker programming}

All the pacemakers in this study were programmed for at least four times: before screening (-4 week), after screening and before randomization (0-week, baseline), 4-week and 12-week follow-up. Before screening, the algorithm was programmed to optimize AF detection, and the function of atrial tachycardia intervention such as post mode switch overdrive pacing, atrial preference pacing and atrial rate stabilization was turned off, which if the pacemaker offered. The detection threshold for AHREs was set to be 180 beats/min over a minimum of 60 secs. Atrial sensitivity was programmed to $0.5 \mathrm{mV}$ with bipolar sensing. Validation of appropriate detection of AHREs or AF was also carried out by scanning atrial electrograms to exclude false-positive detection. The AF burden which was calculated by the hours of all AHREs divided by the hours of follow-up period, the AF with longest duration, and cumulative numbers of AHREs in each visit programming were recorded.

\section{Study endpoints}

The primary endpoint of this study was the change of AF burden after 12-week CRIC. Secondary endpoints were the longest duration of AF and cumulative numbers of AHREs recorded at 4-week and 12week visits.

\section{Statistical analysis}

Data were statistically analyzed with SPSS ver.15.0 (SPSS Inc., Chicago, IL, USA). Continuous variables were expressed as the mean \pm standard deviation, and categorical variables were expressed as the number and percentage (\%). Categorical variables were compared using the $\chi^{2}$ test, and Fisher's exact probability method was used when $T<5$. The normal distribution test was conducted by the Kolmogorov-Smirnov method in the continuous variables, and logarithm transition was done if need. Mean values of clinical characteristics were compared between groups by means of Student's $t$ test,and paired $t$ test were used to compare mean values between baseline and each follow-up for each group. Repeated measurement ANOVA was applied to analyze the effects of time and CRIC on the endpoints of the study including AF burden. A probability value of $p<0.05$ was considered significant.

\section{Results}

A total of 110 patients were screened in this study, and 66 patients ( 32 males and 34 females) were randomized. CRIC was well tolerated during the 12-week period. At last 61 patients completed the study with 31 patients in CRIC group and 30 patients in control. No significant difference was observed between the two groups for follow-up lost rate $(p=1.0)$.

\section{Baseline Characteristics}

Mean age of the 61 patients who completed the whole follow-up was $65.0 \pm 6.0$ years (range: 4975 years). Totally $72 \%$ patients had a history of hypertension, $41 \%$ had -coronary artery disease, $13 \%$ had 
-congestive heart failure, and $21 \%$ had diabetes mellitus. The mean AF burden was $17.3 \% \pm 20.2 \%$, AFepisode was $35.4 \pm 27.9$ per week, and the longest duration of AF was $19.6 \pm 34.0$ hours. The rate of atrial pacing was $58.3 \% \pm 32.4 \%$, and the rate of ventricular pacing was $34.1 \% \pm 42.8 \%$. All the patient characteristics at baseline did not differ between CRIC group and the control group, as shown in Table 1. Types and doses of cardiac medication in this study were not changed for any of the patients during the 16-week period (including 4-week screening period), and the mean doses of $\beta$-blockers, angiotensinconverting enzyme inhibitors or angiotensin receptor blockers, aldosterone receptor antagonists and statins were not different between two groups (Table 1). 
Table 1

Baseline characteristics of study population in CRIC group and control group

\begin{tabular}{|c|c|c|c|}
\hline & Control $(n=30)$ & CRIC $(n=31)$ & p Value \\
\hline Age, years & $65.8 \pm 5.5$ & $64.2 \pm 6.4$ & 0.309 \\
\hline Male(\%)/female(\%) & 16(53.3)/14 (46.7) & 13(41.9)/18(58.1) & 0.373 \\
\hline BMI (kg/m2) & $24.3 \pm 3.2$ & $25.4 \pm 4.0$ & 0.259 \\
\hline Tobacco use(\%) & $5(16.7)$ & $5(16.1)$ & 1.000 \\
\hline Alcohol use(\%) & $4(13.3)$ & $3(9.7)$ & 0.707 \\
\hline $\mathrm{SBP}(\mathrm{mmHg})$ & $125.6 \pm 14.0$ & $130.0 \pm 19.7$ & 0.319 \\
\hline $\mathrm{DBP}(\mathrm{mmHg})$ & $74.2 \pm 11.8$ & $72.0 \pm 12.6$ & 0.492 \\
\hline \multicolumn{4}{|l|}{ Concomitant diseases } \\
\hline Hypertension(\%) & $20(66.7)$ & $24(77.4)$ & 0.349 \\
\hline Coronary artery disease(\%) & $15(50.0)$ & $10(32.3)$ & 0.159 \\
\hline Congestive heart failure(\%) & $5(16.7)$ & $3(9.7)$ & 0.473 \\
\hline Stroke(\%) & 10(33.3) & $10(32.3)$ & 0.929 \\
\hline Diabetes mellitus(\%) & $6(20.0)$ & $7(22.6)$ & 0.806 \\
\hline $\operatorname{COPD}(\%)$ & $3(10.0)$ & $1(3.2)$ & 0.354 \\
\hline \multicolumn{4}{|l|}{ Indication for $\mathrm{PMI}^{\star}$} \\
\hline Sick sinus syndrome(\%) & 25(83.3) & $27(87.1)$ & 0.731 \\
\hline AV Block(\%) & $6(20.0)$ & $7(22.6)$ & 0.806 \\
\hline VP(\%) & $22.7 \pm 38.8$ & $24.0 \pm 40.5$ & 0.905 \\
\hline $\mathrm{AP}(\%)$ & $54.5 \pm 31.2$ & $52.5 \pm 32.0$ & 0.803 \\
\hline
\end{tabular}

*: There are 1 patient suffered from both sick sinus syndrome and AV block in control group and 3 patients in CRIC group.

Data are presented as number (\%) or mean \pm SD.

Abbreviations: ACE, angiotensin-converting enzyme; AP, atrial pacing; ARB, angiotensin receptor blocker; AV Block, atrioventricular block; BMI, body mass index; COPD, chronic obstructive pulmonary disease; DBP, diastolic blood pressure; MRA, aldosterone receptor antagonist; PMI, pacemaker implantation; CRIC, chronic remote ischemic conditioning; SBP, systolic blood pressure; SD, standard deviation; VP, ventricular pacing.

Student's $t$ test or $\chi 2$ test, as appropriate. 


\begin{tabular}{|c|c|c|c|}
\hline & Control $(n=30)$ & CRIC $(n=31)$ & p Value \\
\hline Beta-blockers(\%) & $15(50.0)$ & $21(67.7)$ & 0.159 \\
\hline ACE inhibitors or ARBs(\%) & $12(40.0)$ & 19(61.3) & 0.096 \\
\hline Oral anticoagulant(\%) & 13(43.3) & $9(29.0)$ & 0.245 \\
\hline Antiplatelet agents(\%) & $5(16.7)$ & $9(29.0)$ & 0.251 \\
\hline MRA(\%) & $6(20.0)$ & $7(22.6)$ & 0.806 \\
\hline Statins(\%) & 17(56.7) & $21(67.7)$ & 0.372 \\
\hline \multicolumn{4}{|c|}{$\begin{array}{l}\text { *: There are } 1 \text { patient suffered from both sick sinus syndrome and AV block in control group and } 3 \\
\text { patients in CRIC group. }\end{array}$} \\
\hline \multicolumn{4}{|c|}{ Data are presented as number (\%) or mean \pm SD. } \\
\hline \multicolumn{4}{|c|}{$\begin{array}{l}\text { Abbreviations: ACE, angiotensin-converting enzyme; AP, atrial pacing; ARB, angiotensin receptor } \\
\text { blocker; AV Block, atrioventricular block; BMI, body mass index; COPD, chronic obstructive pulmonary } \\
\text { disease; DBP, diastolic blood pressure; MRA, aldosterone receptor antagonist; PMI, pacemaker } \\
\text { implantation; CRIC, chronic remote ischemic conditioning; SBP, systolic blood pressure; SD, standard } \\
\text { deviation; VP, ventricular pacing. }\end{array}$} \\
\hline Student's $t$ test or $\chi 2$ test, a & te. & & \\
\hline
\end{tabular}

\section{Changes of AF burden}

The burden of AF in CRIC group was decreased significantly at 4-week visit compared with that at 0-week visit $(14.7 \% \pm 18.5 \%$ vs $17.0 \% \pm 20.7 \%, p<0.001)$, which further decreased at 12 -week visit compared with that at 0 -week visit $(8.6 \% \pm 10.2 \%$ vs $17.0 \% \pm 20.7 \%, p<0.001)$ and that at 4 -week visit $(8.6 \% \pm 10.2 \%$ vs $14.7 \% \pm 18.5 \%, p<0.001$ ) (Table 2). There was no difference between each visit point in control group (pष0.05). After 4-week CRIC, the trend of AF burden reduction could be seen in the CRIC group compared with the control group, which did not reach significance $(p=0.337)$. However, AF burden reduced significantly after 12 -week CRIC compared with that in control group $(8.6 \% \pm 10.2 \%$ vs $17.6 \% \pm 19.5 \%, p=$ 0.013). Repeated measurement ANOVA showed that the changes of AF burden was associated with CRIC instead of time $(p<0.01$, data not shown). 
Table 2

Changes in mean geometric atrial fibrillation burden at baseline, week 4 and week 12 .

\begin{tabular}{|c|c|c|c|c|c|c|}
\hline visit points & Ow & $4 w$ & $12 w$ & $\begin{array}{l}P(0 w \text { vs } \\
4 w)\end{array}$ & $\begin{array}{l}P(4 w \text { vs } \\
12 w)\end{array}$ & $\begin{array}{l}P(0 w \text { vs } \\
12 w)\end{array}$ \\
\hline Control group & $\begin{array}{l}17.7 \pm \\
20.0\end{array}$ & $\begin{array}{l}17.5 \pm \\
19.3\end{array}$ & $\begin{array}{l}17.6 \pm \\
19.5\end{array}$ & 0.358 & 0.749 & 0.819 \\
\hline CRIC group & $\begin{array}{l}17.0 \pm \\
20.7\end{array}$ & $\begin{array}{l}14.7 \pm \\
18.5\end{array}$ & $8.6 \pm 10.2$ & $<0.001$ & $<0.001$ & $<0.001$ \\
\hline $\begin{array}{l}\text { P(Cont vs } \\
\text { CRIC) }\end{array}$ & 0.722 & 0.337 & 0.013 & & & \\
\hline \multicolumn{7}{|c|}{ Abbreviations: Cont, control group; CRIC, chronic remote ischemic conditioning. } \\
\hline \multicolumn{7}{|c|}{$\begin{array}{l}\text { Comparison between groups by means of Student's } t \text { test, comparison between baseline and each } \\
\text { follow-up for each group by means of paired t test, and logarithm transition was done before } \\
\text { analysis. }\end{array}$} \\
\hline
\end{tabular}

\section{Changes of AF episodes numbers}

The average number of AF episodes per week in CRIC group was mildly decreased at 4-week visit compared with that at 0 -week visit $(33.6 \pm 26.2$ vs $34.4 \pm 27.0, p=0.189)$, which did not reach significance. However, the mean of AF episodes per week reduced significantly after 12 weeks CRIC compared with that at 0-week $(32.2 \pm 26.2$ vs $34.4 \pm 27.0, p=0.009)$ and that at 4-week $(32.2 \pm 26.2$ vs $33.6 \pm 26.2, p<0.01$ ) (Table 3). There were no differences between each visit point in control group ( $\mathrm{p} \otimes 0.05)$, and between the CRIC group and control group at 4-week $(p=0.827)$ and at 12-week $(p=0.470)$ (Table 3). Repeated measurement ANOVA showed that the changes of AF episodes number were associated with CRIC rather than time $(p<0.05$, data not shown).

Table 3

Changes in numbers of atrial fibrillation episodes per week.

\begin{tabular}{|c|c|c|c|c|c|c|}
\hline visit points & Ow & $4 w$ & $12 w$ & $\begin{array}{l}P(0 w \text { vs } \\
4 w)\end{array}$ & $\begin{array}{l}P(4 w \text { vs } \\
12 w)\end{array}$ & $\begin{array}{l}P(0 w \text { vs } \\
12 w)\end{array}$ \\
\hline Control group & $\begin{array}{l}36.5 \pm \\
29.2\end{array}$ & $\begin{array}{l}36.4 \pm \\
29.6\end{array}$ & $\begin{array}{l}36.9 \pm \\
29.2\end{array}$ & 0.81 & 0.379 & 0.401 \\
\hline CRIC group & $\begin{array}{l}34.4 \pm \\
27.0\end{array}$ & $\begin{array}{l}33.6 \pm \\
26.2\end{array}$ & $\begin{array}{l}32.2 \pm \\
26.2\end{array}$ & 0.189 & 0.009 & $<0.001$ \\
\hline $\begin{array}{l}\mathrm{P} \text { (Cont vs } \\
\text { CRIC) }\end{array}$ & 0.828 & 0.827 & 0.47 & & & \\
\hline \multicolumn{7}{|c|}{ Abbreviations: Cont, control group; CRIC, chronic remote ischemic conditioning. } \\
\hline \multicolumn{7}{|c|}{$\begin{array}{l}\text { Comparison between groups by means of Student's } t \text { test, comparison between baseline and each } \\
\text { follow-up for each group by means of paired t test, and logarithm transition was done before } \\
\text { analysis. }\end{array}$} \\
\hline
\end{tabular}




\section{Changes of longest AF duration}

The longest AF duration at 12-week visit in CRIC group was significantly decreased compared to that at 0 week visit $(17.8 \pm 29.9$ hours vs $20.1 \pm 31.9$ hours, $p<0.001)$ and that at 4 -week visit $(17.8 \pm 29.9$ hours vs $19.1 \pm 29.6$ hours, $p=0.001)$, while 4-week CRIC did not reduce the longest AF duration significantly $(p=$ 0.052) (Table 4). There were no significant differences of longest AF duration among each visit points in control group. The differences between CRIC group and control group at each visit point were also not significant, while repeated measurement ANOVA showed that the changes of longest AF duration were associated with CRIC $(p<0.01$, data not shown).

Table 4

Changes in longest lasting duration (hours) of atrial fibrillation episodes.

\begin{tabular}{|c|c|c|c|c|c|c|}
\hline visit points & Ow & $4 w$ & $12 w$ & $\begin{array}{l}P(0 w \text { vs } \\
4 w)\end{array}$ & $\begin{array}{l}P(4 w \text { vs } \\
12 w)\end{array}$ & $\begin{array}{l}P(0 w \text { vs } \\
12 w)\end{array}$ \\
\hline Control group & $\begin{array}{l}19.1 \pm \\
36.5\end{array}$ & $\begin{array}{l}18.8 \pm \\
35.0\end{array}$ & $\begin{array}{l}19.2 \pm \\
34.2\end{array}$ & 0.471 & 0.218 & 0.873 \\
\hline CRIC group & $\begin{array}{l}20.1 \pm \\
31.9\end{array}$ & $\begin{array}{l}19.1 \pm \\
29.6\end{array}$ & $\begin{array}{l}17.8 \pm \\
29.9\end{array}$ & 0.052 & 0.001 & $<0.001$ \\
\hline $\begin{array}{l}\text { P(Cont vs } \\
\text { CRIC) }\end{array}$ & 0.950 & 0.698 & 0.307 & & & \\
\hline \multicolumn{7}{|c|}{ Abbreviations: Cont, control group; CRIC, chronic remote ischemic conditioning. } \\
\hline \multicolumn{7}{|c|}{$\begin{array}{l}\text { Comparison between groups by means of Student's } t \text { test, comparison between baseline and each } \\
\text { follow-up for each group by means of paired t test, and logarithm transition was done before } \\
\text { analysis. }\end{array}$} \\
\hline
\end{tabular}

\section{Discussion}

The present study has mainly demonstrated that 12-week CRIC can decrease the AF burden in pacemaker-implanted patients, which need to be confirmed in large RCT with long-term follow-up in the future.

$\mathrm{AF}$ is a major risk factor for ischemic stroke and provokes important economic burden along with significant morbidity and mortality, both incidence and prevalence of which have increased over the last 20 years and will continue to increase over the next 30 years ${ }^{20}$. AF burden had not got enough attention for a long time and incorporated into stroke risks scoring systems may partly because of the difficulty to assess these data before. However, with wider usage of different types of CIEDs including pacemakers, ICD, CRT-D/P, and loop recorders, it is easier to monitor long-term atrial arrhythmias and evaluate the AF burden precisely, even if these arrhythmias are asymptomatic and may last only seconds ${ }^{21}$. 
Another reason why AF burden was not valued perhaps is the controversial results of stroke risk between PAF and consistent AF in previous studies. Hart et al ${ }^{22}$ compared 460 intermittent AF participants with 1,552 sustained AF participants during aspirin therapy in the Stroke Prevention in Atrial Fibrillation studies, and found the annualized rate of ischemic stroke in patients with intermittent (3.2\%) was similar to the patients with sustained AF (3.3\%) after a mean of two years follow-up. However, another study drew a distinct conclusion ${ }^{6}$. They analyzed 6563 aspirin-treated patients with AF from the ACTIVEA/AVERROES databases, and found that yearly ischemic stroke rates were $2.1,3.0$, and $4.2 \%$ for paroxysmal, persistent, and permanent $A F$, respectively, with adjusted hazard ratio of $1.83(P<0.001)$ for permanent vs. paroxysmal and $1.44(P=0.02)$ for persistent vs. paroxysmal.

However, AF in those studies was mainly identified with infrequent electrocardiographic techniques ${ }^{22}$, which identified predominantly PAF who are in AF most of the time. As mentioned above, with the wide application of more sensitive and accessible CIED for AF detection, especially the wide usage of DDD pacemakers, we now recognize a much larger prevalence of paroxysmal and short-lasting AF, for which the association with stroke may be different ${ }^{323}$.

Several studies have used CIED to detect AF in different populations and analyzed the association between AF burden and stroke or systemic thromboembolism ${ }^{3}$. Boriani et al performed a pooled analysis of data from 3 prospective studies (PANORAMA, Italian Clinical Services Project, and TRENDS), with 10,016 patients received an CIED implanted, which had at least 3 months of follow-up ${ }^{24}$. After adjustment for CHADS2 score and usage of anticoagulants, Cox regression analysis showed that AF burden was an independent predictor of stroke. The risk for stroke increased by about $3 \%$ for every additional hour increase in the daily maximum of $\mathrm{AF}$ burden ${ }^{24}$. A recent study ${ }^{23}$ found that patients with thromboembolism had higher incidence of pacemaker-detected AF and higher AF burden in the propensity score-matched cohort with comparable CHA2DS2-VASc score, and patients with AF episodes lasting $>6$ min had higher risk of future stroke or thromboembolism on Cox regression analysis (propensity-adjusted HR, 6.75; $P=0.023$ ). Besides thromboembolic events, AF burden was associated with the risk of major adverse cardiovascular events (MACE) ${ }^{25}$. During a median 37.0 months follow-up of 852 patients undergoing CIEDs implantation, the incidence rate (IR) of MACE after AHREs onset was higher in patients developing AHREs $\geq 24 \mathrm{~h}$ (IR 1.13\%/year) than AHREs $\geq 5$ min (IR 0.63\%/year, $p=$ 0.030). Multivariable Cox regression analysis showed that AHREs $\geq 5$ min (HR 1.788, 95\% Cl 1.247$2.562, p=0.002$ ) was interrelated to MACE, and the association was even stronger for AHREs $\geq 24 \mathrm{~h}$ (HR $2.390,95 \% \mathrm{Cl} 1.481-3.857, \mathrm{p}<0.001)$. In our study, AF burden was decreased nearly $50 \%$, about 2 hours per day, by 12 week CRIC ( $\mathrm{p} \otimes 0.001)$. Thus we can speculate that CRIC may be a potential therapy to reduce the risk of ischemic stroke and MACE in these AF patients, while that need to be verified in future studies.

The results of clinical studies associated with CRIC were not always consistent. A potential reason was the different parameters of $\mathrm{CRIC}$, including pressure, duration of deflation and inflation, number of cycles, frequency of CRIC, etc. The total duration of CRIC also differed greatly in many studies, which may play a 
role in the divergent results. We found that 12-week CRIC was more effective than 4-week CRIC in reducing AF load analyzed by repeated measurement ANOVA in our study, which means that CRIC may enhance its effects in a dose-dependent patern ${ }^{15}$. Wei et al demonstrated that preconditioning plus postconditioning every day for 28 days (D1PostC) further attenuated inflammatory and hypertrophic responses, improved cardiac geometry, function, and hemodynamics in rat ischemia/reperfusion injury model compared with perconditioning plus postconditioning every 3 days for 28 days (D-3PostC) ${ }^{26}$. There was also a dose-dependent improvement in survival at 84 days by Kaplan-Meier analysis in separate experiments ${ }^{26}$. Many clinical studies also support that CRIC brings benefits in various conditions, including healing of diabetic foot ulcer ${ }^{27}$, stroke recurrence in patients with symptomatic atherosclerotic intracranial arterial stenosis ${ }^{28}$, and cognitive domains in patients with non-cardiac ischemic stroke ${ }^{29}$.

The mechanism of effects of CRIC on AF has not been fully elucidated. Kosiuk et al ${ }^{17}$ demonstrated that 3 sessions of RIC reduced the inducibility $(23.3 \%$ vs $46.6 \%, p=0.003)$ and sustainability $(15.1 \%$ vs $32.9 \%$, $p=0.01$ ) of PAF significantly, which was possibly mediated by decrease in dispersion of atrial refractory periods $(16.0 \pm 14.0 \mathrm{~ms}$ vs $22.7 \pm 19.0 \mathrm{~ms}, \mathrm{p}=0.21)$ and reduction in atrial conduction delay. Han et al ${ }^{30}$ observed that $\mathrm{AF}$ recurrence in the 3 months after the PAF ablation was significantly lower in the RIC group than in the control group. The RIPC group also showed a lower increase in atrial remodeling marker Matrix Metalloproteinase- 9 and endothelial dysfunction marker von Willebrand-factor in blood samples compared with the control group ${ }^{30}$. Our study has shown that HRV was improved in heart failure patients by CRIC, which was a marker of autonomic nervous stress ${ }^{19}$. As we known, cardiac autonomic nervous stress was closely related to emerging and development of AF. Thus, neural factors may play an important role in CRIC, too. CRIC could regulate immune/ inflammation system ${ }^{14}$ and improve cardiac remodeling ${ }^{31}$, which also plays an important role in the progression of AF. However, the exact mechanism in reduction of $\mathrm{AF}$ burden by $\mathrm{CRIC}$ need to be further exploited in the future.

In conclusion, we found that CRIC can significantly reduce the AF load in patients with pacemaker in a dose-dependent manner. CRIC, a noninvasive and promising adjunctive therapy, thereby may further improve the outcomes over well-established therapies in AF patients.

Limitation: The sample size of this study was relatively small, and the course of follow-up was not long, therefore we have not set mortality and morbidity of stroke as end points. The results of our study and other potential meaningful benefits by CRIC need to be verified in large RCT.

\section{Conclusions}

A 12-week course of CRIC treatment could reduce AF burden in patients with permanent pacemakers, supporting widespread use of CRIC in the daily lives of these patients, which need to be verified in the future. 


\section{List Of Abbreviations}

ACEl: Angiotensin-converting enzyme inhibitor

AF: Atrial fibrillation

AHREs: Atrial high-rate episodes

AP: Atrial pacing

ARB: Angiotensin receptor blocker

AV Block: Atrioventricular block

BMI: Body mass index

BP: Blood pressure

CABG: coronary artery bypass graft

CIEDs: Cardiac implanted electronic devices

COPD: chronic obstructive pulmonary disease

CRIC: Chronic remote ischemic conditioning

DBP: Diastolic blood pressure

HRV: Heart rate variability

IR: Incidence rate

MACE: Major adverse cardiovascular events

MRA: Aldosterone receptor antagonist

PAF: Paroxysmal atrial fibrillation

PMI: Pacemaker implantation

RIC: Remote ischemic conditioning

SBP: Systolic blood pressure

SD: Standard deviation

VP: Ventricular pacing 


\section{Declarations}

Ethics approval and consent to participate: This study was approved by the ethics committee of Zhongda Hospital, Southeast University (Nanjing, China). All the subjects provided a full, informed, written consent before entering the study.

Consent for publication: Not applicable.

Availability of data and materials: The datasets used and/or analysed during the current study are available at http://www.chictr.org.cn/showproj.aspx?proj=21591.

Competing interests: The authors declare that they have no competing interests.

Funding: This work was supported by grant from Nanjing City Development Project of Medical Science and Technology (No. YKK18258), including collection, analysis, and interpretation of data.

Authors' contributions: $C L$ and ZQ designed the study, and CL was a major contributor in writing the manuscript. ZD, YJ and CZ recruited patients, and were responsible for CRIC intervention. YC and LY were in charge of pacemaker programming. $\mathrm{JH}$ and $\mathrm{RL}$ analyzed and interpreted the patient data. All authors read and approved the final manuscript.

Acknowledgements: We would like to thank Professor Hui Jin (School of public health, Southeast University) for his help in statistics.

\section{References}

1. Kirchhof P, Benussi S, Kotecha D, Ahlsson A, Atar D, Casadei B, Castella M, Diener HC, Heidbuchel H, Hendriks $\mathrm{J}$ et al: $2016 \mathrm{ESC}$ Guidelines for the management of atrial fibrillation developed in collaboration with EACTS. Eur Heart J 2016, 37(38):2893-2962. 10.1093/eurheartj/ehw210

2. Ruff CT, Giugliano RP, Braunwald E, Hoffman EB, Deenadayalu N, Ezekowitz MD, Camm AJ, Weitz JI, Lewis BS, Parkhomenko A et al: Comparison of the efficacy and safety of new oral anticoagulants with warfarin in patients with atrial fibrillation: a meta-analysis of randomised trials. Lancet 2014, 383(9921):955-962. 10.1016/S0140-6736(13)62343-0

3. Freedman B, Boriani G, Glotzer TV, Healey JS, Kirchhof P, Potpara TS: Management of atrial high-rate episodes detected by cardiac implanted electronic devices. Nat Rev Cardiol 2017, 14(12):701-714. 10.1038/nrcardio.2017.94

4. January CT, Wann LS, Calkins H, Chen LY, Cigarroa JE, Cleveland JC, Jr., Ellinor PT, Ezekowitz MD, Field ME, Furie KL et al: 2019 AHAVACC/HRS Focused Update of the 2014 AHA/ACC/HRS Guideline for the Management of Patients With Atrial Fibrillation: A Report of the American College of Cardiology/American Heart Association Task Force on Clinical Practice Guidelines and the Heart Rhythm Society in Collaboration With the Society of Thoracic Surgeons. Circulation 2019, 140(2):e125-e151. 10.1161/CIR.0000000000000665 
5. Senoo K, Lip GY, Lane DA, Buller HR, Kotecha D: Residual Risk of Stroke and Death in Anticoagulated Patients According to the Type of Atrial Fibrillation: AMADEUS Trial. Stroke 2015, 46(9):2523-2528. 10.1161/STROKEAHA.115.009487

6. Vanassche T, Lauw MN, Eikelboom JW, Healey JS, Hart RG, Alings M, Avezum A, Diaz R, Hohnloser $\mathrm{SH}$, Lewis BS et al: Risk of ischaemic stroke according to pattern of atrial fibrillation: analysis of 6563 aspirin-treated patients in ACTIVE-A and AVERROES. Eur Heart J 2015, 36(5):281-287a. 10.1093/eurheartj/ehu307

7. Singh JP, Cha YM, Lunati M, Chung E, Li S, Smeets P, O'Donnell D: Real-world behavior of CRT pacing using the AdaptivCRT algorithm on patient outcomes: effect on mortality and atrial fibrillation incidence. J Cardiovasc Electrophysiol 2020. 10.1111/jce.14376

8. Przyklenk K, Bauer B, Ovize M, Kloner RA, Whittaker P: Regional ischemic 'preconditioning' protects remote virgin myocardium from subsequent sustained coronary occlusion. Circulation 1993, 87(3):893-899. 10.1161/01.cir.87.3.893

9. Kharbanda RK, Mortensen UM, White PA, Kristiansen SB, Schmidt MR, Hoschtitzky JA, Vogel M, Sorensen K, Redington AN, MacAllister R: Transient limb ischemia induces remote ischemic preconditioning in vivo. Circulation 2002, 106(23):2881-2883. 10.1161/01.cir.0000043806.51912.9b

10. Heusch G, Botker HE, Przyklenk K, Redington A, Yellon D: Remote ischemic conditioning. J Am Coll Cardiol 2015, 65(2):177-195. 10.1016/j.jacc.2014.10.031

11. Botker HE, Kharbanda R, Schmidt MR, Bottcher M, Kaltoft AK, Terkelsen CJ, Munk K, Andersen NH, Hansen TM, Trautner S et al: Remote ischaemic conditioning before hospital admission, as a complement to angioplasty, and effect on myocardial salvage in patients with acute myocardial infarction: a randomised trial. Lancet 2010, 375(9716):727-734. 10.1016/S0140-6736(09)62001-8

12. Sloth AD, Schmidt MR, Munk K, Kharbanda RK, Redington AN, Schmidt M, Pedersen L, Sorensen HT, Botker HE, Investigators C: Improved long-term clinical outcomes in patients with ST-elevation myocardial infarction undergoing remote ischaemic conditioning as an adjunct to primary percutaneous coronary intervention. Eur Heart J 2014, 35(3):168-175. 10.1093/eurheartj/eht369

13. Xie J, Zhang X, Xu J, Zhang Z, Klingensmith NJ, Liu S, Pan C, Yang Y, Qiu H: Effect of Remote Ischemic Preconditioning on Outcomes in Adult Cardiac Surgery: A Systematic Review and Metaanalysis of Randomized Controlled Studies. Anesth Analg 2018, 127(1):30-38.

10.1213/ANE.0000000000002674

14. Zhao W, Li S, Ren C, Meng R, Ji X: Chronic Remote Ischemic Conditioning May Mimic Regular Exercise:Perspective from Clinical Studies. Aging Dis 2018, 9(1):165-171. 10.14336/AD.2017.1015

15. Thijssen DH, Maxwell J, Green DJ, Cable NT, Jones H: Repeated ischaemic preconditioning: a novel therapeutic intervention and potential underlying mechanisms. Exp Physiol 2016, 101(6):677-692. 10.1113/EP085566

16. Candilio L, Malik A, Ariti C, Barnard M, Di Salvo C, Lawrence D, Hayward M, Yap J, Roberts N, Sheikh A et al: Effect of remote ischaemic preconditioning on clinical outcomes in patients undergoing cardiac 
bypass surgery: a randomised controlled clinical trial. Heart 2015, 101(3):185-192. 10.1136/heartjnl2014-306178

17. Kosiuk J, Langenhan K, Stegmann C, Uhe T, Dagres N, Dinov B, Kircher S, Richter S, Sommer P, Bertagnolli $L$ et al: Effect of remote ischemic preconditioning on electrophysiological parameters in nonvalvular paroxysmal atrial fibrillation: The RIPPAF Randomized Clinical Trial. Heart Rhythm 2020, 17(1):3-9. 10.1016/j.hrthm.2019.07.026

18. Chen L, Zhou Q, Jin H, Zhu K, Zhi H, Chen Z, Ma G: Effects of Remote Ischaemic Conditioning on Heart Rate Variability and Cardiac Function in Patients With Mild Ischaemic Heart Failure. Heart Lung Circ 2018, 27(4):477-483. 10.1016/j.hlc.2017.03.164

19. Taralov ZZ, Terziyski KV, Kostianev SS: Heart Rate Variability as a Method for Assessment of the Autonomic Nervous System and the Adaptations to Different Physiological and Pathological Conditions. Folia Med (Plovdiv) 2015, 57(3-4):173-180. 10.1515/folmed-2015-0036

20. Lippi G, Sanchis-Gomar F, Cervellin G: Global epidemiology of atrial fibrillation: An increasing epidemic and public health challenge. Int J Stroke 2020:1747493019897870.

$10.1177 / 1747493019897870$

21. Healey JS, Connolly SJ, Gold MR, Israel CW, Van Gelder IC, Capucci A, Lau CP, Fain E, Yang S, Bailleul C et al: Subclinical atrial fibrillation and the risk of stroke. N Engl J Med 2012, 366(2):120-129. 10.1056/NEJMoa1105575

22. Hart RG, Pearce LA, Rothbart RM, McAnulty JH, Asinger RW, Halperin JL: Stroke with intermittent atrial fibrillation: incidence and predictors during aspirin therapy. Stroke Prevention in Atrial Fibrillation Investigators. J Am Coll Cardiol 2000, 35(1):183-187. 10.1016/s0735-1097(99)00489-1

23. Chu SY, Jiang J, Wang YL, Sheng QH, Zhou J, Ding YS: Pacemaker-detected atrial fibrillation burden and risk of ischemic stroke or thromboembolic events-A cohort study. Heart Lung 2020, 49(1):66-72. 10.1016/j.hrtlng.2019.07.007

24. Boriani G, Glotzer TV, Santini M, West TM, De Melis M, Sepsi M, Gasparini M, Lewalter T, Camm JA, Singer DE: Device-detected atrial fibrillation and risk for stroke: an analysis of $>10,000$ patients from the SOS AF project (Stroke preventiOn Strategies based on Atrial Fibrillation information from implanted devices). Eur Heart J 2014, 35(8):508-516. 10.1093/eurheartj/eht491

25. Pastori D, Miyazawa K, Li Y, Szekely O, Shahid F, Farcomeni A, Lip GYH: Atrial high-rate episodes and risk of major adverse cardiovascular events in patients with cardiac implantable electronic devices. Clin Res Cardiol 2020, 109(1):96-102. 10.1007/s00392-019-01493-z

26. Wei M, Xin P, Li S, Tao J, Li Y, Li J, Liu M, Li J, Zhu W, Redington AN: Repeated remote ischemic postconditioning protects against adverse left ventricular remodeling and improves survival in a rat model of myocardial infarction. Circ Res 2011, 108(10):1220-1225.

10.1161/CIRCRESAHA.110.236190

27. Shaked G, Czeiger D, Abu Arar A, Katz T, Harman-Boehm I, Sebbag G: Intermittent cycles of remote ischemic preconditioning augment diabetic foot ulcer healing. Wound Repair Regen 2015, 23(2):191196. 10.1111/wrr.12269 
28. Meng R, Asmaro K, Meng L, Liu Y, Ma C, Xi C, Li G, Ren C, Luo Y, Ling F et al: Upper limb ischemic preconditioning prevents recurrent stroke in intracranial arterial stenosis. Neurology 2012, 79(18):1853-1861. 10.1212/WNL.0b013e318271f76a

29. Feng $X$, Huang L, Wang Z, Wang L, Du X, Wang Q, Xue S: Efficacy of remote limb ischemic conditioning on poststroke cognitive impairment. J Integr Neurosci 2019, 18(4):377-385. 10.31083/j.jin.2019.04.1192

30. Han R, Liu X, Zheng M, Zhao R, Liu X, Yin X, Liu X, Tian Y, Shi L, Sun K et al: Effect of remote ischemic preconditioning on left atrial remodeling and prothrombotic response after radiofrequency catheter ablation for atrial fibrillation. Pacing Clin Electrophysio/ 2018, 41(3):246-254. 10.1111/pace.13271

31. Yamaguchi T, Izumi Y, Nakamura Y, Yamazaki T, Shiota M, Sano S, Tanaka M, Osada-Oka M, Shimada K, Miura $\mathrm{K}$ et al: Repeated remote ischemic conditioning attenuates left ventricular remodeling via exosome-mediated intercellular communication on chronic heart failure after myocardial infarction. Int J Cardio/ 2015, 178:239-246. 10.1016/j.ijcard.2014.10.144

\section{Figures}

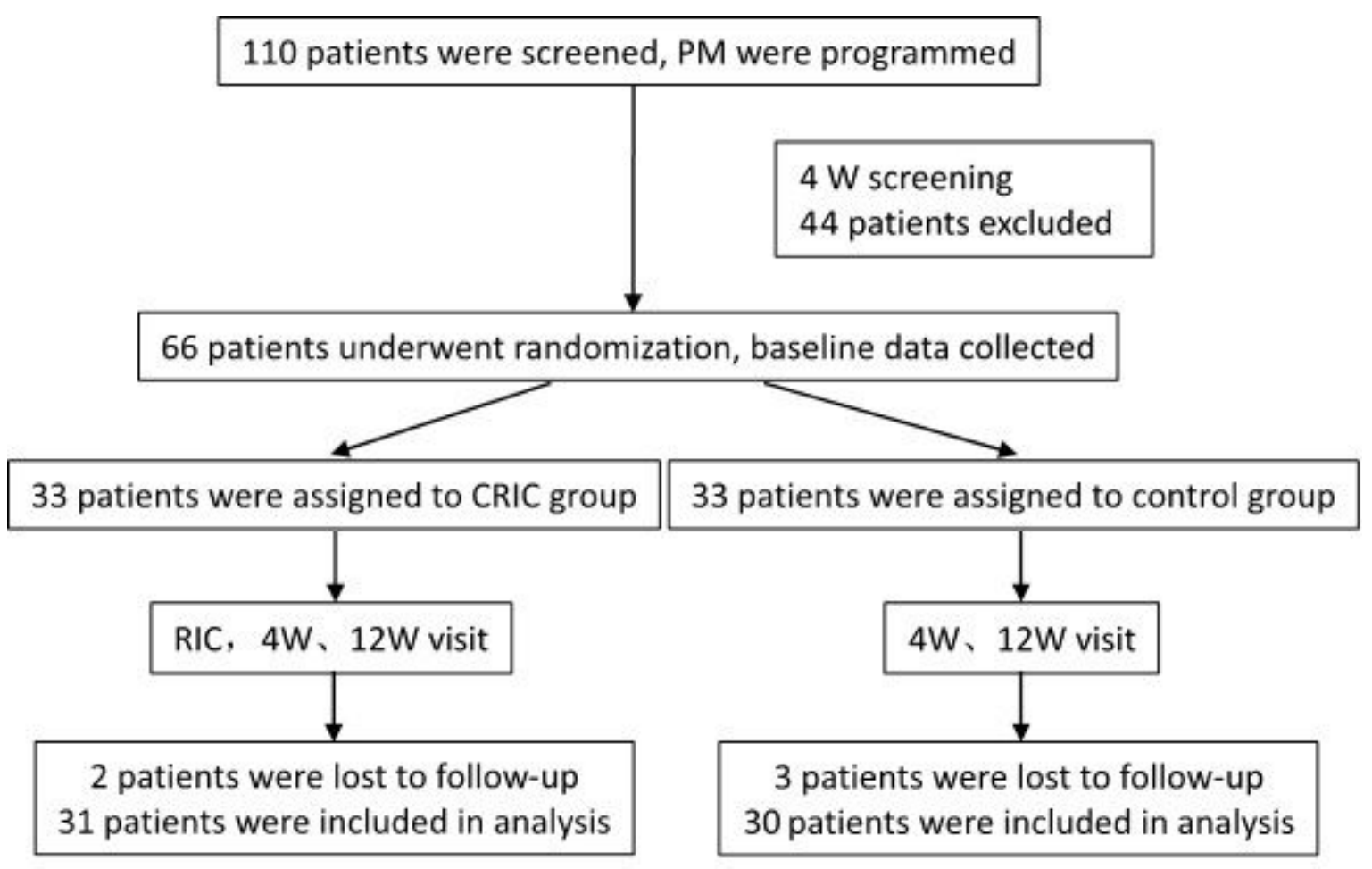

Figure 1

Flow chat of the study. Abbreviations: PM, pacemaker; CRIC, chronic remote ischemic conditioning; W, week.

\section{Supplementary Files}


This is a list of supplementary files associated with this preprint. Click to download.

- CONSORT2010Checklist.doc 